\title{
Case report: atypical presentation of Mycobacterium tuberculosis uveitis preceding nodular scleritis
}

\author{
Sunee Chansangpetch*, Anita Manassakorn, Prasart Laksanaphuk and Usanee Reinprayoon
}

\begin{abstract}
Background: Intraocular tuberculosis is uncommon and has various clinical presentations. Lack of specific clinical clues can make the diagnosis challenging. The purpose of this study is to report a clinical presentation of tuberculous iridocyclitis that mimics phacolytic glaucoma and has a distinctive inflammatory deposit in the inner side of the cornea. This report is the first to describe the progression of tuberculous iridocyclitis to nodular scleritis without evidence for extraocular tuberculous infection.
\end{abstract}

Case presentation: A 78-year-old, immunocompetent woman presented with subacute intraocular inflammation with high intraocular pressure, mimicking phacolytic glaucoma. Distinct pigment keratic precipitates were noted on the first visit. Even though the cataract extraction was uneventful and adequate anti-inflammatory drugs were given, the inflammation did not subside as expected. Seven weeks later, she developed two scleral abscesses, which were subsequently explored for microbiological investigation. The smears of the pus revealed positive acid-fast bacilli stain and PCR for Mycobacterium tuberculosis complex. Eventually, the pus culture grew Mycobacterium tuberculosis. Antituberculosis medications were prescribed. After 1 month of treatment, the abscesses were cured. However, her visual acuity did not improve at the last visit.

Conclusions: This case revealed an unusual presentation and untreated course of tuberculosis iridocyclitis. Pattern of keratic precipitates may indicate the presence of tuberculosis.

Keywords: Mycobacterium tuberculosis, Uveitis, Nodular scleritis, Phacolytic glaucoma, Intraocular inflammation

\section{Background}

Most Mycobacterium tuberculosis infection involves the lungs which is known as pulmonary tuberculosis. However, tuberculosis can also occur in other places of the body. For example, approximately $15-20 \%$ of immunocompetent patients [1] and $50 \%$ of immunocompromised patients [2] can experience extrapulmonary tuberculosis which can affect the lymphatic system, bone, gastrointestinal system, central nervous system, genitourinary system, cardiovascular system, and the skin.

\footnotetext{
* Correspondence: bae_sunnee@yahoo.co.uk

This paper was presented at the 30th Asia-Pacific Academy of

Ophthalmology Congress on 1-4 April, 2015, at Guangzhou, China.

Department of Ophthalmology, Faculty of Medicine, Chulalongkorn

University and King Chulalongkorn Memorial Hospital, 1873 Rama 4 Road, Pathumwan, Bangkok 10330, Thailand
}

Ciomed Central

(c) 2015 Chansangpetch et al. Open Access This article is distributed under the terms of the Creative Commons Attribution 4.0 International License (http://creativecommons.org/licenses/by/4.0/), which permits unrestricted use, distribution, and reproduction in any medium, provided you give appropriate credit to the original author(s) and the source, provide a link to the Creative Commons license, and indicate if changes were made. The Creative Commons Public Domain Dedication waiver (http://creativecommons.org/publicdomain/zero/1.0/) applies to the data made available in this article, unless otherwise stated. eye but this is rare. The incidence of ocular tuberculosis varies among series of cases. In endemic areas such as Southeast Asia, many immunocompromised HIV patients have extrapulmonary tuberculosis [3]. Usually, the ophthalmic involvement of Mycobacterium tuberculosis is secondary from hematogenous spreading. Primary infection is seldomly reported [4] but can occur at many sites such as the orbit, lacrimal gland, eyelid, conjunctiva, sclera, iris, choroid, retina, and optic nerve, and have a broad range of clinical manifestations $[4,5]$.

Tuberculous infection of the eyes can present with various clinical symptoms and frequently mimic other ophthalmic diseases. Thus, these patients are often misdiagnosed [6]. Many of the challenges of diagnosing tuberculosis in the eye are encountered such as limited amount of specimen available for testing, lack 
of laboratory investigation, poor yield from intraocular specimen, and the absence of uniformity in its diagnostic criteria [7]. Delayed diagnosis can result in severe visual impairment as well as the loss of the globe.

One of the most common ocular clinical manifestations is the posterior uveitis with choroidal tubercles $[5,6]$ whereas only $0.6-7.9 \%$ of anterior uveitis or iridocyclitis is caused by tuberculosis $[8,9]$. As a result of this, we report the clinical presentation of tuberculous iridocyclitis that mimics phacolytic glaucoma and have distinct deposits in the inner side of the cornea known as keratic precipitates (KP). Moreover, we also describe the clinical progression from uveitis to nodular scleritis in immunocompetent patient which has never been reported elsewhere.

\section{Case presentation}

A 78-year-old Thai female presented with a right painful ocular irritation and progressive blurring of the vision for 2 months. At the first visit, best-corrected visual acuity was hand motion for the right eye and 20/70 for the left eye. The intraocular pressure was $40 \mathrm{mmHg}$ for the right eye and $10 \mathrm{mmHg}$ for the left eye. On slit lamp examination, her right eye had marked ciliary injection, mild corneal edema with inferior heavily pigmented KP, cells grade $3+$, and flares grade $3+$ in the anterior chamber. The pupil was mid-dilated, fixed, and did not react to light. The lens was swollen and totally opaque (mature cataract) (Fig. 1). The left eye was unremarkable except for senile immature cataract. She was diagnosed with phacolytic glaucoma of the right eye and underwent extracapsular cataract extraction and intraocular lens (IOL) implantation on the next day without intraoperative complication. In early post-operative period, the intraocular pressure was well controlled without anti-glaucoma medication and the visual acuity slightly improved to 20/200. Topical antibiotic and steroid eye drops were prescribed but the inflammation in the anterior segment of the eye did not subside.
Seven weeks later, her right eye developed 2 yellowish scleral masses at 5 and 11 o'clock (Fig. 2). The inflammation continued to persist (cell and flare grade $3+$ ) and fundus could not be examined due to vitreous haze grade 3 . Ultrasound of the right eye showed minimal retrolental vitreous opacity and the vitreous echoes showed rapid after movements. The retinal-choroidal thickness was $0.68 \mathrm{~mm}$. Post-operative endophthalmitis was suspected and the patient was sent for vitreous tapping and intravitreous injection with vancomycin and ceftazidime. The culture for aerobic, anaerobic, and fungus revealed all negative results. Topical fortified cefazolin and ceftazidime eye drops were given. When topical steroid was discontinued, this resulted in worsening of the inflammation in the anterior chamber and scleral masses. Because the masses increased in size and showed obvious abscess formation, exploration and debridement of the scleral abscesses were done. Specimen was sent for Gram stain, $\mathrm{KOH}$ test, aerobic bacteria, anaerobic bacteria, and fungus cultures, all of which were negative.

One week after scleral exploration, her eye was still severely inflamed with corneal edema, cell grade 4+, flare grade $3+$, and frank fibrin in front of the IOL. The anterior chamber became shallower and resulted in captured IOL. New scleral abscess was noted at 11 o'clock adjacent to the explored area. The clinical signs were getting worse and did not respond to broad-spectrum fortified antibiotic eye drops. Scleral exploration with abscess debridement was performed for the second time. The authors then became highly suspicious that she may have atypical pathogens so the specimen was also sent for acid-fast bacilli (AFB) and modified AFB stain. The smear showed AFB positive organisms (Fig. 3).

Real-time polymerase chain reaction (PCR) for Mycobacterium tuberculosis complex was done on the scleral abscess, aqueous humor, and vitreous, which were positive in the abscess and aqueous. The tests of the vitreous samplings yielded negative results. Later, the pus culture

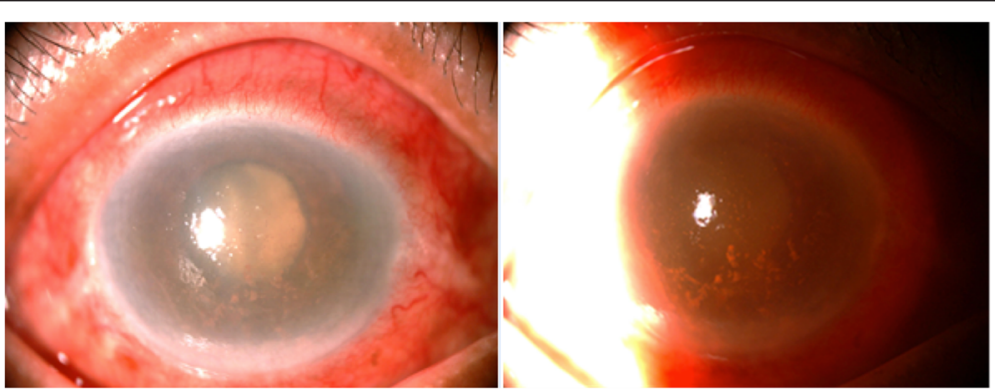

Fig. 1 Slit lamp examination of the right eye. (picture shown on the left side) Diffuse illumination showed markedly ciliary injection, corneal edema, and swollen lens. (picture shown on the right side) The sclerotic scatter technique could detect the inferiorly dispersed pigmented keratic precipitates. 

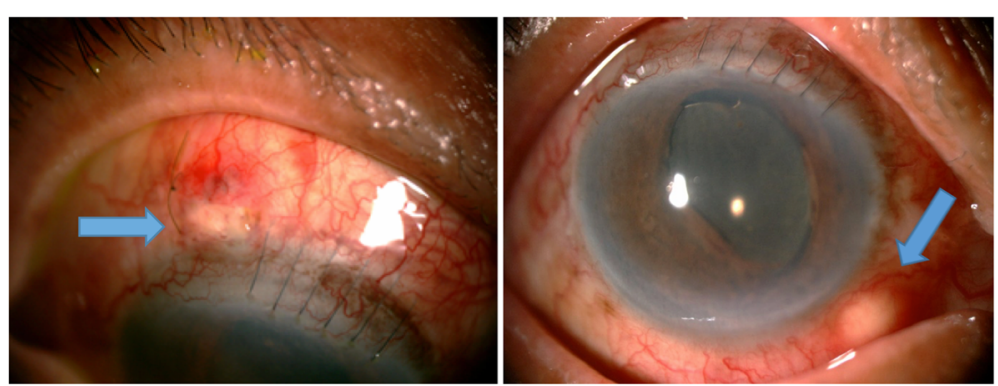

Fig. 2 Two yellowish scleral abscesses (indicated by an arrow). An abscess of $2.5 \mathrm{~mm}$ in diameter at 11 o'clock, superior to the wound of the extracapsular cataract extraction where the sutures have been removed next to the abscess (in the picture shown on the left side). The other abscess of $2 \mathrm{~mm}$ in diameter located at 5 o'clock (in the picture shown on the right side)

with a combination of liquid (Mycobacteria growth indicator tube) and solid media (Ogawa) grew Mycobacterium tuberculosis.

The patient was referred to an infectious disease specialist to investigate for systemic tuberculosis. She reported a loss of $10 \mathrm{~kg}$ of body weight in the past 3 months. She denied fever, night sweats and chest symptoms. Physical examination including her left eye was otherwise normal. The hematocrit was $35.3 \%$ (reference range $36-45 \%$ in women). The white blood cell count was 6500 cells $/ \mathrm{mm}^{3}$ and the cells were predominantly neutrophils $(70 \%)$. The other routine laboratory test results were normal. A chest radiograph revealed normal finding. Sputum AFB was also negative. Neither the evidence of extraocular tuberculosis nor history of tuberculosis contact was identified.

The patient was then started on systemic antituberculosis medications with isoniazid $250 \mathrm{mg} / \mathrm{d}$, rifampicin $450 \mathrm{mg} / \mathrm{d}$, ethambutal $1000 \mathrm{mg} / \mathrm{d}$ and pyrazinamide $1250 \mathrm{mg} / \mathrm{d}$. Prednisolone acetate eyedrop was prescribed after starting anti-tuberculosis for a week. A 4-drug anti-tuberculosis regimen was continued for the first 2 months, and for the next 4 months, only isoniazid and rifampicin were used. Regular follow-ups were performed. After 1 month of treatment, no new abscess was found and the intraocular inflammation subsided a bit even though her visual acuity was still hand motion. After 2 months of treatment, the inflammation had completely subsided with no development of new abscess and the IOP was controlled but the vision did not perceive any light so the authors speculated that this was due to the ischemia of the anterior segment.

\section{Discussion}

In our case, at the first visit, she was misdiagnosed as having phacolytic glaucoma because she had a mature cataract and elevated intraocular pressure. However, the persistent post-operative inflammation and progressive scleral abscesses made us reconsider the possibility that the origin of the disease may be infectious, especially an atypical one. Apart from the chronic post-operative endophthalmitis, atypical infectious pathogens such as Nocardia spp. and Mycobacterium spp. initially were included in our differential diagnosis. Endophthalmitis was later ruled out by negative bacteria and fungus culture results. Moreover, the location of vitreous haze was localized only in the retrolental area instead of the vitreous chamber and the normal retinal-choroidal thickness from ultrasonography suggested that the vitreous cells were the reaction to a marked anterior segment inflammation rather than endophthalmitis, a vitreous infection in origin. Our initial differential diagnosis was

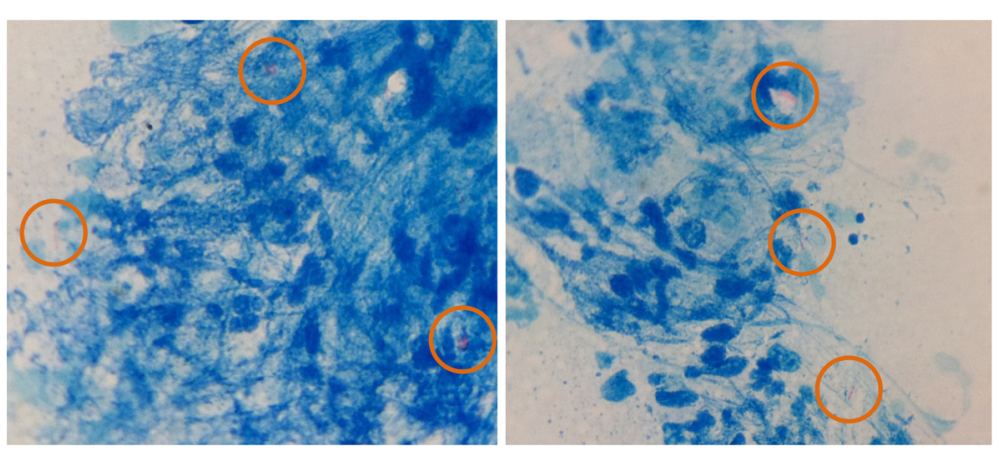

Fig. 3 Photomicrograph of the red, short curved, acid fast rod organisms in AFB stain 
Nontuberculous mycobacterium because a prolonged steroid use and prior ocular surgery were identified as the risk factors for developing this group of mycobacterium especially the rapid growers [10]. However, when the culture results came back as Mycobacterium tuberculosis, her past clinical course started to make sense.

It should be noted that cataract can frequently develop in the eyes followed by chronic or recurrent uveitis [11]. Severe intraocular inflammation can raise the intraocular pressure due to either the blockage of the intertrabecular space by inflammatory cells or edema of the trabecular meshwork which is the main route for aqueous drainage. The prolonged course of inflammation can permanently damage these meshwork functions. In our case, the patient seemed to have the uveitis for a long period prior to visiting the ophthalmology clinic. These two clinical manifestations, a consequence of a long-run untreated uveitis as aforementioned, misled us to diagnose the patient with phacolytic glaucoma because she had a cataract with high intraocular pressure.

In general phcolytic cases, after cataract surgery, the inflammation should rapidly subside in the first month because the lens, which are the cause of the inflammation, have been removed. Even though the inflammation persisted to 7-8 weeks, it should have been very low grade. However, for this patient, the inflammation subsided a bit in the first week after the cataract surgery when compared to the pre-operative state, but continued to persist at a moderate to high grade throughout the next 7 weeks. Thus, we believe that this inflammation was caused by a pre-existing infection rather than the secondary one.

There are a few cases of intraocular tuberculosis presented with anterior uveitis [6]. Presentation usually includes granuloma of the iris or inflammatory cells at the angle of the anterior chamber previously described as mutton fat KP. Its characteristics are greasy clumps of inflammatory cells at the posterior cornea [6]. This type of KP indicates that it is a granulomatous inflammation, which can also be found in toxoplasmosis and sarcoidosis [10]. However, for this patient, the characteristics of the typical mutton fat KP were absent. Instead, the KPs were heavily pigmented and inferiorly located. The presence of KPs in this patient probably was an important sign that was overlooked which indicated that the etiology was uveitis rather than phacolytic glaucoma. Therefore, we suggest that differential diagnosis of tuberculous uveitis must be kept in mind despite of the absence of a typical mutton fat KP, especially when a prominent pigment is observed.

Mycobacterium tuberculosis can also cause scleritis but this is rare [12]. The anterior part of the sclera is affected more frequently than the posterior. Examination may reveal necrotizing nodular scleritis along with scleral ulceration [5]. Kesen, et al. [13] reported, in 2009, a case of atypical drug-resistant tuberculous scleritis presented with masses at the anterior sclera. The masses subsequently progressed to a large area of necrotic sclera. Damodaran, et al. [14] also reported, in 2012, a case with severe intraocular inflammation and a large mass lesion in the globe detected by ultrasonography. The patient did not respond to anti-inflammatory or immunosuppressive agents resulting in perforation of the eye. In both cases, the diagnoses of tuberculous scleritis were confirmed afterward by histopathology of the enucleated eyes.

As far as we are aware, this is the first report that demonstrates the progression of untreated Mycobacterium tuberculosis uveitis to scleral abscesses. The progression can be the course of the disease itself when there is a migration of an increasing number of organisms into the adjacent structure, from the intraocular to sclera. It is also possible that the scleral involvement may have been the consequence of a direct inoculation of the organisms during the cataract surgery which could have spread the mycobacterium-filled aqueous to the sclera.

Diagnostic tests for tuberculosis in endemic area are very different compared to non-endemic areas. For example, the tuberculin skin tests and interferon-gamma release assays may be useful for diagnosing tuberculosis in non-endemic area, but the use of these tests are limited and cannot differentiate between the latent tuberculous infection and disease in endemic area such as Thailand $[5,6,15]$. Therefore, in order to diagnose tuberculosis disease in an endemic country, Mycobacterium tuberculosis culture, the gold standard, is needed to detect ocular tuberculosis even though the sensitivity of the culture is low for the detection of paucibacillary infection in which case a molecular technique may be helpful bearing in mind the limitation of false-positive results [6].

\section{Conclusion}

In conclusion, this case emphasizes the need to be aware of ocular tuberculosis in an endemic area especially with unusual presentations and prolonged clinical course of ocular inflammation. A high index of suspicion of tuberculosis would require clinical examination aided by an appropriate microbiological testing because it can help in early diagnosis and management of the infection. The pattern of KPs may indicate the presence of tuberculous infection.

\section{Consent}

Written informed consent was obtained from the patient for publication of the case report and any accompanying images. A copy of the written consent is available for review by the Editor of this journal. 


\begin{abstract}
Abbreviations
KP: Keratic precipitate; IOL: Intraocular lens; AFB: Acid-fast bacilli;

PCR: Polymerase chain reaction.
\end{abstract}

\title{
Competing interests
}

The authors declare that they have no competing interests.

\section{Authors' contributions}

UR was significantly involved in the patient's treatment including operation and follow-up, supplied the clinical data, contributed important intellectual content and revised the manuscript. SC had a major role in the laboratory investigation, data collection, drafting, and revision of the manuscript. AM and PL contributed significantly to critically revising the manuscript for important intellectual content. All authors participated in the review and final approval of the manuscript.

\section{Acknowledgements}

We would like to thank Dr. Wimonrat Prateepasen and Dr. Supawat Trepatchayakorn for their kind helps in providing important clinical data. We also thank Suthidee Petsong, our faculty's medical technologist for the information regarding mycobacterium laboratory investigation techniques.

Received: 8 July 2015 Accepted: 15 October 2015

\section{Published online: 28 October 2015}

\section{References}

1. Sharma SK, Mohan A. Extrapulmonary tuberculosis. Indian J Med Res. 2004;120(4):316-53.

2. Golden MP, Vikram HR. Extrapulmonary tuberculosis: an overview. Am Fam Physician. 2005;72(9):1761-8.

3. Tognon MS, Fiscon M, Mirabelli P, Graziani G, Peracchi M, Sattin A, et al. Tuberculosis of the eye in Italy: a forgotten extrapulmonary localization. Infection. 2014;42(2):335-42. doi:10.1007/s15010-013-0554-4.

4. Tabbara KF. Tuberculosis. Curr Opin Ophthalmol. 2007;18(6):493-501. doi:10.1097/ICU.0b013e3282f06d2e.

5. Bodaghi B, LeHoang P. Ocular tuberculosis. Curr Opin Ophthalmol. 2000;11(6):443-8

6. Gupta V, Gupta A, Rao NA. Intraocular tuberculosis-an update. Surv Ophthalmol. 2007:52(6):561-87. doi:10.1016/.jsurvophthal.2007.08.015.

7. Patel SS, Saraiya NV, Tessler HH, Goldstein DA. Mycobacterial ocular inflammation: delay in diagnosis and other factors impacting morbidity. JAMA ophthalmol. 2013;131(6):752-8. doi:10.1001/jamaophthalmol.2013.71.

8. Rodriguez A, Calonge M, Pedroza-Seres M, Akova YA, Messmer EM, D'Amico DJ, et al. Referral patterns of uveitis in a tertiary eye care center. Arch Ophthalmol. 1996;114(5):593-9.

9. Singh R, Gupta V, Gupta A. Pattern of uveitis in a referral eye clinic in north India. Indian J Ophthalmol. 2004;52(2):121-5.

10. Girgis DO, Karp CL, Miller D. Ocular infections caused by non-tuberculous mycobacteria: update on epidemiology and management. Clin Experiment Ophthalmol. 2012;40(5):467-75. doi:10.1111/j.1442-9071.2011.02679.x.

11. Secchi AG. Cataracts in Uveitis. Trans Ophthalmol Soc U K. 1982;102(Pt 3):390-4.

12. Yeh S, Sen HN, Colyer M, Zapor M, Wroblewski K. Update on ocular tuberculosis. Curr Opin Ophthalmol. 2012;23(6):551-6. doi:10.1097/ ICU.0b013e328358ba01.

13. Kesen MR, Edward DP, Rao NA, Sugar J, Tessler HH, Goldstein DA. Atypical infectious nodular scleritis. Arch Ophthalmol. 2009;127(8):1079-80. doi:10.1001/archophthalmol.2009.197.

14. Damodaran K, George AE, Goel S, Khetan V, Noronha V, Biswas J. Tubercular sclerouveitis masquerading as an ocular tumor: a case report. Ocul Immunol Inflamm. 2012;20(5):368-71. doi:10.3109/09273948.2012.708957.

15. Sudharshan S, Ganesh SK, Balu G, Mahalakshmi B, Therese LK, Madhavan HN, et al. Utility of QuantiFERON(R)-TB Gold test in diagnosis and management of suspected tubercular uveitis in India. Int Ophthalmol. 2012;32(3):217-23. doi:10.1007/s10792-012-9554-0.

\section{Submit your next manuscript to BioMed Central and take full advantage of:}

- Convenient online submission

- Thorough peer review

- No space constraints or color figure charges

- Immediate publication on acceptance

- Inclusion in PubMed, CAS, Scopus and Google Scholar

- Research which is freely available for redistribution

Submit your manuscript at www.biomedcentral.com/submit 